\title{
Optical filters with fractal transmission spectra based on diffractive optics
}

\author{
Omel Mendoza-Yero, ${ }^{1, *}$ Gladys Mínguez-Vega, ${ }^{1}$ Mercedes Fernández-Alonso, ${ }^{1}$ Jesús Lancis, ${ }^{1}$ \\ Enrique Tajahuerce, ${ }^{1}$ Vicent Climent, ${ }^{1}$ and Juan A. Monsoriu ${ }^{2}$ \\ ${ }^{1}$ Departament de Física, GROC-UJI, Universitat Jaume I, 12080 Castelló, Spain \\ ${ }^{2}$ Departamento de Física Aplicada, Universidad Politécnica de Valencia, E-46022 Valencia, Spain \\ *Corresponding author: omendoza@uji.es
}

Received November 3, 2008; accepted December 24, 2008;

posted January 21, 2009 (Doc. ID 103645); published February 19, 2009

\begin{abstract}
The duality between the axial irradiance distribution originated by any circularly symmetric diffracting aperture under monochromatic illumination and its diffracted spectral intensity at a fixed on-axis point under broadband illumination is highlighted and experimentally investigated. Two applications are derived from this basic result. On the one hand, we suggest the use of a broadband source and a spectrometer for a singleshot measurement of the axial response of pupil filters. Second, we implement a spectral filter having a transmission spectrum with a fractal structure of frequencies. Experimental results and potential applications in synthetic spectra designs are provided. (C) 2009 Optical Society of America

OCIS codes: 260.1960, 050.1940, 300.6190.
\end{abstract}

The potential of the Fresnel zone plate (FZP) to shape the electromagnetic wavefront has emerged during the past few years in different fields of optics. Most of the applications are based on its diffractive nature, which allows for light bending without the use of refractive components unsuitable for certain regions of the electromagnetic spectrum. The dispersive properties of the FZP were exploited as early as in the 1970s to implement spectroscopic measurements [1,2]. More recently, this basic device has been applied for carrying out time-resolved x-ray absorption spectroscopy and pump-probe experiments [3]. In combination with the coherent radiation coming from a femtosecond laser, the zone plate spectrometer has been proposed as a cost-effective method to slice the broadband spectrum of the ultrashort pulse [4]. A similar diffractive optics layout was implemented for the spectral selection of a single femtosecond high-order harmonic generated in a rare gas medium [5].

Wavelength splitting by broadband-illuminated FZPs is the underlying physical mechanism for all of the above applications. However, the spatial splitting of a monochromatic plane wave into different focal points along the optical axis is perhaps the best known feature of the diffractive FZP [6]. This intriguing similarity stems from a more general principle that has its origin in the dual role played by the wavelength $\lambda$ and the axial distance $z$ from the aperture in the Huygens-Fresnel diffraction formula. In the case of a simple diffractive lens, one-to-one mapping between $\lambda$ and $z$ allows for achieving, i.e., spectral matched filtering under broadband LEDs illumination [7].

In this Letter we theoretically formulate, and experimentally verify, the above duality within the paraxial regime. For verification purposes, we use a femtosecond laser as a broadband source and a conventional fiber spectrometer for single-shot monitoring the field diffracted by a generalized zone plate (GZP). This element shows a circularly symmetric binary transmittance, periodic in the squared radial co- ordinate, with period $p^{2}$. The ratio between the areas of the whole period and of its transparent part is a positive integer number $\varepsilon$. The periodic nature of this pupil ensures a focal structure for the axial response, which permits a high signal-to-noise ratio spectral measurement around the focal positions. This allows for transferring results between the uncorrelated fields of diffractive optics and synthetic spectra. In this direction, we suggest the use of pupil filters with well-known axial response [8] to tailor the energy spectrum of a broadband source. We demonstrate diffractive optics-based spectral filters that exhibit spectrum scalability. It means that within the spectral profile of the filter some of its parts have the same shape as the whole spectrum. The use of diffractive optics to shape the spectra was first recognized by Sinclair et al. in [9].

To formulate the duality, we consider a circularly symmetric mask, with wavelength-independent amplitude transmittance $t(r)$, which is illuminated by a plane wave that has an initial broad spectrum $S_{0}(\lambda)$. The variable $r$ stands for the radial coordinate at the input plate. The irradiance distribution at any transversal plane located at a distance $z$ from the mask, $I(z, R)$, is assessed by the Huygens-Fresnel diffraction integral expressed in a cylindrical coordinate system as

$$
I(z, R)=\int I(z, R, \lambda) \mathrm{d} \lambda,
$$

where the spectral irradiance, $I(z, R, \lambda)$, is given by

$$
\begin{aligned}
I(z, R, \lambda)= & S_{0}(\lambda) \\
& \times\left|\frac{2 \pi}{i \lambda z} \int_{0}^{\infty} t(r) J_{0}\left(\frac{2 \pi}{\lambda z} R r\right) \exp \left[i \frac{\pi}{\lambda z} r^{2}\right] r \mathrm{~d} r\right|^{2} \\
= & S_{0}(\lambda) M(z, R, \lambda) .
\end{aligned}
$$

In Eq. (2), $R$ is the radial coordinate at the output plane, the term $M(z, R, \lambda)$ is the so-called spectral modifier function, and $J_{0}(x)$ denotes the Bessel func- 
tion of the first kind with order zero and argument $x$. Initially, we assume $R=0$; that is, we restrict to the intensity along the optical axis. A further simplification can be done if we make the change of variable $\mu=r^{2}$ in Eq. (1). Now, $M(z, 0, \lambda)$ is evaluated as the scaled Fourier transform of the transmittance $t(\mu)$. The scale factor is $v=1 /(2 \lambda z)$.

For a fixed value of $z$, the function $M(z, 0, \lambda)$ can be interpreted as a spectral filter that transforms the initial spectrum $S_{0}(\lambda)$ into a modified one. In this case, $I(z, 0, \lambda)$ reads as the spectral irradiance. Also for a single monochromatic component $\lambda$, the function $M(z, 0, \lambda)$ provides the quasi-monochromatic axial response of the diffracting mask. Note the dual role played by the variables $z$ and $\lambda$ in the modifier function as they provide an identical functional dependence. This is the basis for the axial-spectral irradiance duality. From a practical point of view, the axial irradiance profile for the monochromatic channel $\lambda$ $=\lambda_{0}$ within the axial interval $\Delta z$ ranging from $z_{\text {ini }}$ to $z_{\text {end }}$ is identical to the profile of the spectral irradiance at the axial point $z=z^{*}$ within the spectral band $\Delta \lambda$ of the polychromatic source determined by the extreme wavelengths $\lambda_{\text {ini }}=\lambda_{0} z_{\text {ini }} / z^{*}$ to $\lambda_{\text {end }}=\lambda_{0} z_{\text {end }} / z^{*}$. Strictly speaking, the axial-spectral duality holds only for a flat spectrum source $\left[S_{0}(\lambda)=\right.$ constant $]$. However, once the function $S_{0}(\lambda)$ is known, the spectral data can be weighted by the amount $1 / S_{0}(\lambda)$ so that the duality remains valid.

We verified experimentally the above duality by use of a GZP as a diffracting mask. For clarity, a GZP consists of $Q$ concentric transparent rings with an equal area onto an opaque screen. The axial irradiance distribution generated by this aperture under monochromatic illumination of wavelength $\lambda=\lambda_{0}$ was extensively studied in [10]. In this way, we found

$$
M(z, 0, \lambda)=\sin ^{2}\left(\frac{\pi p^{2}}{2 \varepsilon \lambda z}\right) \frac{\sin ^{2}\left(\frac{Q \pi p^{2}}{2 \lambda z}\right)}{\sin ^{2}\left(\frac{\pi p^{2}}{2 \lambda z}\right)} .
$$

Axial focal positions, the local maxima of $M(z, 0, \lambda)$, are located at points $z_{n}=p^{2} /\left(2 \lambda_{0} n\right)$, with $n$ a positive integer. Exceptions occur when $n=j \varepsilon$ (with $j=1,2, \ldots$ ), because for the above positions the slowly oscillating envelope vanishes. The axial irradiance $M(z, 0, \lambda)$ consists of a characteristic profile that repeats along the optical axis at different positions and with a different scale. This profile is restricted to the range $\left[z_{n=j \varepsilon}, z_{n=(j-1) \varepsilon}\right]$, for any value of $j \neq 1$, and shows the presence of $(\varepsilon-1)$ modulated foci. From a practical point of view it is important to recognize that the width of the range $\Delta z=z_{n=(j-1) \varepsilon}-z_{n=j \varepsilon}=p^{2} / 2 \lambda_{0} \varepsilon j(j-1)$ does depend on the choice of $j$.

To test the axial-spectral irradiance duality, we experimentally measure the irradiance profile by using a femtosecond laser and a spectrometer. The GZP was constructed by direct laser writing on a chrome photomask with the help of a photolithographic machine. The laser writing machine allows us to obtain

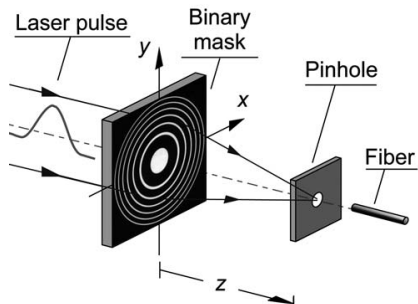

(a)

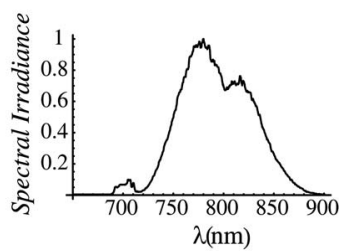

(b)
Fig. 1. (a) Optical device for single-shot monitoring the axial irradiance distribution generated by the binary pupils. (b) Spectral irradiance of the incident femtosecond pulse.

photomasks with a global resolution of $0.2 \mu \mathrm{m}$ and a minimum feature size of $0.8 \mu \mathrm{m}$. For our experiment we chose $p=4 \mathrm{~mm}, \varepsilon=4$, and the radius of the mask was $a=1 \mathrm{~cm}$. The optical system is shown in Fig. 1(a). The light coming from a Ti:sapphire laser impinges perpendicularly on the mask. The central wavelength of the femtosecond pulse is $\lambda_{0}=780 \mathrm{~nm}$. The broad spectrum of the pulse is plotted in Fig. 1(b). For the measurement, we use a fiber optics spectrometer. A pinhole with radius $r_{0}=5 \mu \mathrm{m}$ was located in front of the fiber to select only the contributions of the field in the vicinity of the optical axis. From a practical point of view it is advisable to locate the spectrometer at a distance $z^{*}$ that coincides with a focal position of the mask. In addition, a proper choice of the integer $j$ must be done to fit the axial range $\Delta z=z_{n=(j-1) \varepsilon}$ $-z_{n=j \varepsilon}$ within the broad spectrum of the source $\Delta \lambda$ $=300 \mathrm{~nm}$. For our experiment $j=4$, as the measurements were done at the focus $n=14$. Results of the experiment are shown in the top of Fig. 2(a) after weighting the nonflat initial source spectrum. We show how the measured spectrum fits nicely the theoretical plot of the axial irradiance $M(z, 0, \lambda)$, which is also shown in the bottom of Fig. 2(a). In fact, the correlation coefficient between both curves gives a similarity of $86 \%$.

The duality applies to more general rotationally invariant pupils. This opens the door for single-shot monitoring the axial response of pupil filters commonly employed in imaging devices by use of a broadband, either coherent or incoherent, source and commercial spectrometers.

It is well known that optical fields derived from the diffraction of monochromatic waves by fractal structures show a characteristic fractal on-axis irradiance profile [11]. Based on the established duality, here we implement optical filters that show a transmission spectrum with a fractal distribution of wavelengths. Transmission properties of fractal Cantor multilayer dielectric structures also show spectra scalability as demonstrated by Lavrinenko et al. [12]. Our approach, however, is radically different, as it is based on the use of diffractive optical elements.

The key is to use a fractal GZP $[13,14]$. For simplicity, we choose the corresponding fractal GZP resulting from the suppression of some transparent rings of the above GZP. The generation process of fractal GZP from a Cantor set is controlled and characterized by three fundamental parameters: the generator $2 N-1$, 

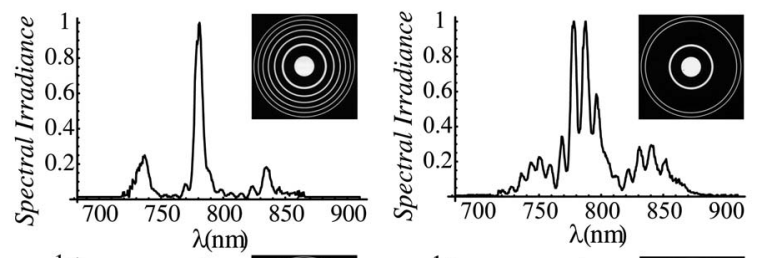

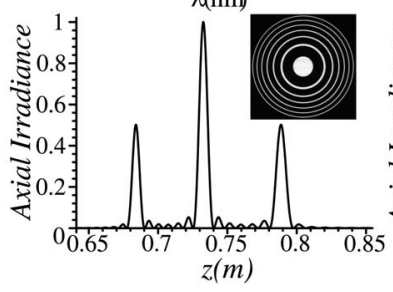

(a)

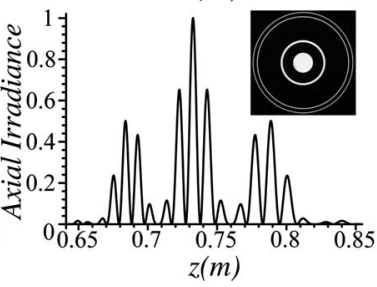

(b)
Fig. 2. Measured spectral irradiance for (a) GZP (top) and for (b) corresponding fractal GZP (top). The axial irradiance distribution for (a) GZP predicted by Eq. (3) (bottom) and for (b) corresponding fractal GZP predicted by Eq. (4) (bottom). Scaled versions of the binary pupils are given by the insets.

the growing stage $S$, and the opening ratio $\varepsilon$. In Fig. 2 (b) we show as insets a scaled version of the resulting fractal GZP derived from the triadic Cantor set $(N=2)$ for the second stage of growing $S=2$ and $\varepsilon=4$.

Again, the monochromatic axial irradiance $M\left(z, 0, \lambda_{0}\right)$ generated by the fractal GZP consists of a characteristic profile with a focal structure that repeats along the optical axis. In [14], we demonstrated the self-similarity property for the monochromatic axial irradiance distribution generated by the above set of masks.

In accordance with the axial-spectral duality, the above results are readily transferred to the spectral domain thus obtaining spectra scalability. The measured output spectrum for a pinhole radius $r_{0}$ $=5 \mu \mathrm{m}$ of the spectrometer is shown in the top of Fig. 2(b). The spectrometer was located at the same axial position as before because the corresponding fractal GZP also has a focus there. The theoretical monochromatic axial irradiance for the central wavelength of the pulse is plotted in the bottom of Fig. 2(b) with the help of the expression [14]

$$
\begin{aligned}
M(u, 0, \lambda)= & 4 \sin ^{2}\left(\frac{\pi u}{(\varepsilon N-(\varepsilon-1))^{S}}\right) \\
& \times \prod_{i=1}^{S} \frac{\sin ^{2}\left(N \frac{\varepsilon \pi u}{(\varepsilon N-(\varepsilon-1))^{i}}\right)}{\sin ^{2}\left(\frac{\varepsilon \pi u}{(\varepsilon N-(\varepsilon-1))^{i}}\right)} .
\end{aligned}
$$

In Eq. (4) the variable $u=a^{2} /(2 \lambda z)$. A nice fit between the above plots, this time with a correlation coefficient of $67 \%$, is obtained. The scalability property for the spectrum follows directly from the self-similar feature of the axial irradiance profile reported in [14]. The case in the top of Fig. 2(b) represents, to the best our knowledge, the first attempt to transfer into the spectrum domain the fractal irradiance properties of fractal diffractive optical elements.

In summary we experimentally show how to get the on-axis irradiance shape of a circularly symmetric binary pupil by means of a simple spectral measurement. The proved irradiance-to-spectrum link is useful to design filters based on tailoring the axial irradiance distribution of circular binary pupils. Finally, an optical filter with a fractal transmission spectrum is reported based on the fractal GZP.

This research was funded by the Spanish Ministerio de Educación y Ciencia (MEC), Spain, through Consolider Programme Science and Applications of Ultrafast Ultraintense Lasers (SAUUL) CSD200700013. The authors are very grateful to the Serveis Centrals d'Intrumentació Científica (SCIC) of the Universitat Jaume I for the use of the Ti:sapphire femtosecond laser and the laser writing machine.

\section{References}

1. P. N. Keating, R. K. Mueller, and T. Sawatari, J. Opt. Soc. Am. 62, 945 (1972).

2. A. Fedotowsky and K. Lehovec, Appl. Opt. 16, 582 (1977).

3. U. Vogt, M. Wieland, T. Wilhein, M. Beck, and H. Stiel, Rev. Sci. Instrum. 72, 53 (2001).

4. A. Gürtler, J. J. Gilijamse, A. A. R. Wetzels, L. D. Noordam, E. Sali, and M. Bellini, Opt. Commun. 270, 336 (2007).

5. J. Gaudin, S. Rehbein, P. Guttmann, S. Godé, G. Schneider, Ph. Wernet, and W. Eberhardt, J. Appl. Phys. 104, 033112 (2008).

6. E. Hecht and A. Zajac, Optics (Addison-Wesley, 1987), p. 445.

7. S. Sinzinger, V. Arrizón, and J. Jahns, Proc. SPIE 3002, 186 (1997)

8. M. T. Caballero, P. Andrés, A. Pons, J. Lancis, and M. Martínez-Corral, Opt. Commun. 246, 313 (2005).

9. M. B. Sinclair, M. A. Butler, S. H. Kravitz, W. J. Zubrzycki, and A. J. Ricco, Opt. Lett. 22, 1036 (2008).

10. O. Mendoza-Yero, G. Mínguez-Vega, J. Lancis, and V. Climent, J. Opt. Soc. Am. A 24, 3600 (2007).

11. C. Allain and M. Coiltre, Phys. Rev. B 33, 3566 (1986).

12. A. V. Lavrinenko, S. V. Zhukovsky, K. S. Sandomirski, and S. V. Gaponenko, Phys. Rev. E 65, 036621 (2002).

13. G. Saavedra, W. D. Furlan, and J. A. Monsoriu, Opt. Lett. 28, 971 (2003).

14. O. Mendoza-Yero, M. Fernández-Alonso, G. MínguezVega, J. Lancis, V. Climent, and J. A. Monsoriu, arXiv.org e-Print archive, arXiv:0811.0343v1, November 3, 2008, http://arXiv.org/abs/0811.0343. 